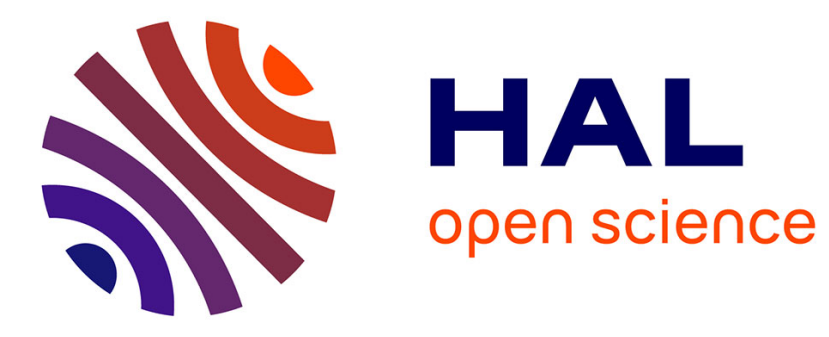

\title{
Why study turn-taking sequences in interspecies interactions?
}

Chloé Mondémé

\section{To cite this version:}

Chloé Mondémé. Why study turn-taking sequences in interspecies interactions?. Journal for the Theory of Social Behaviour, 2022, 52 (1), pp.67-85. 10.1111/jtsb.12295 . hal-03228856

\section{HAL Id: hal-03228856 https://hal.science/hal-03228856}

Submitted on 23 Nov 2021

HAL is a multi-disciplinary open access archive for the deposit and dissemination of scientific research documents, whether they are published or not. The documents may come from teaching and research institutions in France or abroad, or from public or private research centers.
L'archive ouverte pluridisciplinaire HAL, est destinée au dépôt et à la diffusion de documents scientifiques de niveau recherche, publiés ou non, émanant des établissements d'enseignement et de recherche français ou étrangers, des laboratoires publics ou privés. 


\section{Why study turn-taking sequences in interspecies interactions?}

Chloé Mondémé, CNRS (French National Center for Scientific Research)

École Normale Supérieure de Lyon, France

Institutional address:

ENS de Lyon, 15 parvis René Descartes, 69342 Lyon Cedex 07

chloe.mondeme@ens-lyon.fr

ORCID: 0000-0002-5927-4650

Abstract. The "turn-taking" system, a notion referring to the dynamics of verbal exchanges in face-to-face interaction, is often presented as the hallmark of human language (Levinson, 2016). However, recent works have investigated turn-taking mechanisms in several nonhuman taxa, thus referring to the moment-by-moment coordinated alternation of actions or vocalizations, for instance in primates or birds. These findings have been interpreted as evidence of elementary forms of sequence organization in the animal world, and therefore as evidences of an evolutionary pathway, leading from very rough forms of signal alternation, to the complex multimodal and conversational turn-taking system used by humans. This article first reviews and discusses these findings. It then makes the case for going beyond the study of uniquely intra-specific forms of communication and interaction. It argues that considering interspecies interaction is of interest for social sciences in general, and linguistics scholarship in particular, at least in two respects. First, on an empirical level, to investigate a widespread phenomenon, namely ordinary human-animal interactions, as it occurs mostly in domestic settings - a topic that has been relatively neglected in the study of social interaction so far (for a range of reasons that we shall touch on). Secondly, on a more speculative level, it assumes that ordinary interspecies interactions, especially as they unfold with domestic pets or wild animals in natural settings ( $v s$. in experimental settings), are a valuable locus to explore rudimentary forms of sequence organization, that are neither governed by linguistic and cultural norms, nor entirely the product of stereotypical species-related behavioral patterns. We posit that fundamental principles of sociality are to be found in the contextual adjustments of multimodal actions and in the emergence of meaning in interspecies interactions.
Keywords. Interspecific interaction; turn-taking; origins of language; cooperation; multimodal communication; conversation analysis

\section{Introduction}

Since the beginning of the 2000s, numerous attempts to explore the limits of human uniqueness have emerged, including through an investigation of one of humans' most intriguing capacities: language and cooperative forms of communication. One parameter in particular has been scrutinized: turn-taking and its correlate, sequential organization. Originally coined as systematic 'mechanisms' (Sacks, Schegloff, Jefferson, 1974), and sometimes referred to as 'universals' (Stivers et al., 2009; Kendrick et al., 2020) or 'universal infrastructure' (Pika et al., 2018), turn-taking and conversational mechanisms are treated as analytical entry points to open the black box of communication.

The concept of turn-taking was first developed to account for the orderly distribution of speakership within human conversation (Sacks et al., 1974; Sacks, 1992; Schegloff, 2007). Although counter-intuitively (for it only dealt with the properties of human unique capacity of articulate verbal language), this notion has recently been brought, and appropriated, by the field of animal communication. The existence of rulegoverned conversation-like mechanisms has since been investigated in a vast array of animal species: in birds and nonhuman primates, of course, but also in many mammals such as elephants, whales, dolphins and bats (see Pougnault, 2020)

There are a variety of reasons why it is of interest to study rule-governed communicational exchanges and turn-taking mechanisms in the animal kingdom. The most obvious perhaps, and the one that has kept linguists, anthropologists and psychologists busy for decades - and continues to do so - is the access it may offer to the 
'origins of language'. Building on the idea that the evolution of interlocutive exchanges could have paved the way for more complex forms of communication and cooperation, it is thought that investigating the dynamics of turn-taking in primate communication will help to reconstruct the steps that have progressively led to human language. The second reason is rooted in the inherently cooperative dimension of dyadic exchanges: exploring the way individuals of a same species alternate their actions could be a window on more elaborated forms of sociality and cooperative behaviors, addressing issues of joint action, collective behaviors and shared intentions.

In this article, after briefly reviewing these hypotheses, I make the case for the study of sequential organization in interspecies human-animal interaction. In order to build bridges between knowledges emerging from the field of linguistics (and focusing primarily on human conversation) and the fields of biology and behavioral sciences (mostly focusing on animal behavior), I argue that interspecies interactions are a valuable locus to access and investigate rudimentary forms of sequential organizations that are neither completely the product of cultural norms (although they might embed forms of normativity), nor stereotypical responses of an organism to the behavior of a conspecific. Two species need to adjust to one another, admittedly building on the particularities of their species-typical behavioral repertoires, but procedurally trying to make sense out of it, thus creating emergent ways of interacting and acting together.

On this topic maybe more than on any other, disciplinary boundaries are not clear cut. There has been a constant import-export of concepts travelling back and forth between philosophy of mind to primatology and social psychology, comparing the empirical data, and enriching the theoretical frameworks. For instance, the famous concept of 'theory of mind', now commonly used in philosophy of mind and social cognition, stemmed from primatology and originally referred to non-human primates' socio-cognitive skills (chimpanzees, in the seminal 1970 paper by Premack and Woodruff) and their ability to make predictions about the state of mind of their conspecifics. It then spread throughout all the disciplines of the mind and was reintroduced into developmental psychology. The notion was thus adopted by Wimmer and Perner (1983) in their 'false-belief task' experiments with children. The trajectory of this concept illustrates the porosity between disciplines interested in the study of human and animal behavior.

Without reducing the findings of social sciences to those of natural sciences, and trying to avoid naive epistemological comparisons, this article aims to explore the trajectory of the concept of turn-taking, and see its relevance for describing interspecific interactions. It provides an overview of the different attempts to identify forms of rulegoverned conversational mechanisms in the animal world, showing how this has been used to explore two different, though complementary, issues: the origin(s) of human language on the one hand, and the evolution of cooperation and sociality on the other. It then argues for developing analyses of interspecies interactions, whose heuristics may help to grasp the rudimentary forms of communication in social interaction. Finally, while reporting on empirical examples of interspecific human-animal sequences of action, it aims to address an epistemological question: is turn-taking more than just a coordinated alternation of actions? If so, what insights does this specifically bring to an understanding of human action? We assume that studying turn-taking mechanisms in interspecies contexts may not only shed light on the origins of language as a highly elaborate form of cooperative exchange, but it may also help to pinpoint the fundamentally reflexive dimension of human social interaction. 
1. Turn-taking in animals: an investigation of the origins of language and on the evolution of cooperation

In recent years, there have been many attempts to identify a communicational pattern, referred to as 'turn-taking', in several different taxa. The description of turn-taking mechanisms in animals is not solely intended to explore the distinctive features of a particular species. It has a broader scope, which is to further the understanding of the evolution of human language and orientation towards (ultra)sociality (Richerson \& Boyd, 1998). This section proposes an overview of these recent works, and considers their claims about the origins of language and the roots of cooperation.

\subsection{Sequence organization and the origins of human language}

The question of the existence of proto-conversations in animal species is not completely new. Back in the 1970 's, along with the boom of sociobiology, the animal world became a model for describing human society, but the reverse was also true. The vocabulary of human affairs was imported to the study of animal behavior. Some primatologists studying antiphonal calls in monkeys even analyzed the phenomenon as a 'conversation' (Snowdon \& Cleveland, 1984), drawing on the psychologists Duncan and Fiske's early descriptions of "dynamic patterning in conversation" (1979) in humans. More recently, multimodal communicational exchanges in non-human primates have also been investigated through a conversational lens (Rossano, 2013; Frölich et al., 2017; Pika et al. 2018).

This examination of rule-governed conversational exchanges in animal species is not solely driven by a desire to explore species-typical communicational abilities. It may also aim to provide a better understanding of human communication, in term of its evolutionary emergence and development. The argument is made that investigating the dynamics of turn-taking in animal communication will help to reconstruct the steps that have progressively led to human language. The idea of a phylogenetical continuity in the primate clade is at the root of the comparison between what may be found in the actual pan species (chimpanzees, bonobos) and the hypothetical social behavior of our common ancestor, from whom we diverged about 8 million years ago. This assumption is used to support the analysis of conversational forms of communicational exchanges in different animal species.

Communicational animal behavior is mostly described by using the vocabulary of human affairs: be it with the analogy of conversation, or with the analogy of music or orchestration (e.g., 'duetting', 'chorusing'). We will refer to these two analogical models as the conversational model and the orchestral model and briefly review how they analytically call on the notion of turn-taking to explore the origins of human language. It should be noted that the goal here is not to give an exhaustive survey of every linguisticlike rule-governed property identified in the animal world (see e.g. Schlenker et al., 2017; Zuberbühler, 2005; for reviews of semantical, syntactical and pragmatic properties), but only those properties that have been described in terms of turn-taking.

The orchestral model

Animal vocal signaling has always been intriguing, including for the early naturalists and animal behaviorists (see Crist, 1999; Burkhardt, 2005). This is especially true for bird species, whose vocal signals, as they are produced in turns, were easily described with the metaphor of orchestration ("duetting", "chorus singing", "countersinging", etc.).

Bird vocal signals are often produced in coordination or in alternation: 'duetting' involves a series of calls with typical temporal association, 'chorus singing' is a given call type produced by several individuals in overlap, and 'countersinging' is a response to a neighboring territorial songbird. In addition to an extensive body of research in avian vocal interaction, practices of duetting have also been documented in primates (e.g. 
Deputte, 1982, for gibbons), and chorusing has been well investigated in Pan species (Fedureck \& Slocombe, 2013). This musical, or orchestral, model progressively became a springboard for the conversational model. In 1984, the psychologists Snowdon \& Cleveland described "conversations among pygmy marmosets" (a variety of New World monkeys), focusing in particular on antiphonal calls produced by one member of the group in response to another for territorial issues or to maintain social bonds. Without advancing any hypothesis regarding how this evolved, the authors observed that these calls are governed by a conversational 'rule system' (1984: 15) and concluded by appealing for further study: "There is no evidence for sequential dependencies as well in the calling between animals. It will be of great interest to analyze the patterns of calling in other species of primate to determine if such 'conversations' appear elsewhere" (1984: 20).

In a 2000 study, Todt and Naguib studied vocal duels in passerine birds. Significantly, the article is subtitled "The use of song as a model of communication", and the metaphor thus becomes a 'model'. Although they do not clearly describe a mechanism resembling a turn-taking system in passerines, they nevertheless identify conversational rules quite similar to those observed by the first analysts of social interaction (Goffman,

1981) and human conversation (Sacks et al., 1974; Goodwin, 1981). In their words:

« Participating animals often respond to each other's signals in very specific ways and each of the interacting individuals usually takes the role of both a signaler and a listener » (Todt \& Naguib, 2000, p. 247)

They go on to state that the temporal change in roles "can be described as "turn-taking"” (2000: 286). Other authors would take this further, positing that animal duetting can be of interest for the evolution of communicative behavior in general: "Avian duetting has the potential to be a model system for the ontogeny of vocal interaction" (Logue \& Krupp,
2016).

All these studies report strict alternation of calls. Yet turn-taking here refers to a somehow stereotyped temporal alternation of calling, rather than a display of social rules. The extent to which these rules, or regularities, approach a human turn-taking system as conceptualized seminally by analysts of social interaction is still largely under investigated (Pika et al., 2018).

\section{The conversational model}

As mentioned above, some researchers went even further than the orchestral model by explicitly making reference to 'conversation'. The metaphor or analogy made between animal vocal alternation and dialogue (Todt \& Naguib, 2000) is even more relevant for our purpose here. The conversational model emphasizes how turn-taking mechanisms unfold in vocal or gestural units. What distinguishes strict vocal alternation from its interpretation as a dialogue is the greater degree of flexibility between signaler and listener: when "signals are related to each other in a dialogue-like manner" (Todt \& Naguib, 2000, p. 248), this directly implies a greater flexibility to the contingencies. In the animal behavior literature, turn-taking is usually summarized as a set of rules, directly drawn (as curious as this may be) from the seminal paper by Sacks, Schegloff and Jefferson (1974): (i) one party speaks at a time; (ii) the order for speaker turn is variable; (iii) gaps and overlaps are minimized.

Essentially, human conversation serves as a matrix for understanding the distribution of vocal communication between individual animals. Such rule-governed organizational properties have been reported in the vocal interactions of primates (Masataka, 2003), meerkats (Demartsev et al., 2018), bonobos (Levrero et al., 2019), and gorillas (Pougnault et al., 2020), as well as in birds (Henry et al., 2015). In particular, 
these studies report phenomena of overlap avoidance and interlocutor selectivity rules (Levrero et al., 2019).

In addition, gestural forms of turn-taking are now extensively documented, essentially in great apes (Slocombe, Waller, Liebal, 2011; Rossano, 2013; Fröhlich, 2017; inter alia.), which is not surprising, as gesture plays an increasing role in the theories of language origins. The 'vocal theory of language origins' (according to which calls represents a precursor of human language) has long opposed the 'gestural theory', a dichotomy that now tends to be dialectically bridged through by the 'multimodal theory of language origins' (see Frölich et al., 2019 for a proposition, and Prieur et al., 2020, for a recent overview).

To sum-up this section, the turn-taking machinery provides a model for the description of vocal or gestural communicational exchanges in the animal reign that is anchored in the description of human cultural practices: music, orchestration, conversation (as it features a distribution of turns). Its investigation rests on the assumption, still hypothesized at this stage (Levinson \& Holler, 2014; Pika et al., 2018) that there is a phylogenetical inheritance of rudimentary forms of vocal/gestural alternation in more complex forms of turn-taking.

\subsection{Turn-taking in animals: sociality and cooperation among conspecifics}

A second rationale for the examination of turn-taking in different animal species is that turn-taking mechanisms can be the by-product (or the precursor, depending on the hypothesis) of elaborated forms of cooperation. The relationship between turn-taking mechanisms, the emergence of language and the evolution of cooperative behavior is deeply intertwined. In this section, we explore a number of studies that have drawn links between the analysis of behavior in terms of cooperation and two analytical categories pertaining to turn-taking, i.e. sequential dependencies, and recipient design matters.
From probabilistic constraints to "sequential dependencies"

At the beginning of the 1960s, in the framework of a project on simian sociology, zoologist Stuart Altmann studied the social behavior and communication of rhesus monkeys, starting from the following observation:

"The behavior of an individual depends upon preceding actions within the individual's interacting group. Such antecedent events do not, however, completely determine present behavior. Rather, probabilistic constraints exist between events within a sequence of actions. Such sequential dependencies between the actions of group members indicate that social communication is taking place » (Altmann, 1965: 490)

He therefore decided to use stochastic models that would represent the sequence of events as probabilities, to see the constraints that a behavior $\mathrm{X}$ has upon a behavior $\mathrm{Y}$. This mathematical method makes predictions about subsequent behaviors depending on a given 'antecedent' (for example related to patterns of attacks and threats, or friendly behaviors such as grooming)

Interestingly, Altmann's argument of probabilistic constraints in primates influenced early thinkers of the turn-taking machinery, in particular Duncan (1972) whose paper entitled - significantly - "Some signals and rules for taking speaking turns in conversation" also explicitly refers to Goffman (1964) - evidencing the apparent absence of a gap, in the 1960's and 1970's, between the analysis of animal behaviors and human conducts. From this point, starts the shift from stochastic reasoning to a comprehensive demonstration of mutual and accountable adjustments in interaction. Interestingly, Duncan's paper itself appears to be an influential reading for the elaboration of the "simplest systematics" (and is mentioned in a footnote).

One crucial contribution of conversation analysis (CA) is to claim that utterances are both context-shaped and context-renewing (Heritage, 1984) or in other words, that 
turns have a prospective and a retrospective orientation (Goodwin, 2006). This sensitivity to the context (see Duranti \& Goodwin, 1992) is therefore a feature of the cooperative dimension of social interaction. The analysis of sequential implicativeness, as elaborated by CA (see below) - and in contrast to the probabilistic calculation of antecedent/consequent turns - might allow for a characterization in terms of cooperation. Pedersen (2016) inspired by Sacks' work, claims that such phenomena are clearly visible in interactions with bonobos.

Sensitivity to the attentional state and "recipient design" matters

Directly stemming from the previous point, is the sensitivity exhibited by a 'speaker', or rather a 'signaler' to his/her audience, and the way he/she calibrates the utterance accordingly. This has been referred to as 'recipient design' - or the sensitivity to particular others, and classically described by Sacks et al. (1974) as follows:

"By 'recipient design' we refer to a multitude of respects in which the talk by a party in a conversation is constructed or designed in ways which display an orientation and sensitivity to the particular other(s) who are co-participants.” (1974, p. 727)

Other studies in CA (Schegloff, 1972, Hutchby, 1995; inter alia) have extended the concept to encompass the presumed knowledge states of their recipients. But without going thus far, and without requiring shared knowledge of the epistemic statuses of the interactants, studies in animal behavior have focused on how features of gestural or vocal signals are designed for a particular audience (again, for examples within chimpanzee and bonobo mother/infant dyads see Fröhlich et al., 2016a; or Rossano \& Liebal, 2014).

The question of knowing to whom an utterance or a signal is addressed is absolutely crucial to determine whether a given vocal or gestural display was a stereotyped emission of a communicative ethological behavior or a more context- sensitive production of information, adjusted to its potential interlocutors. Links between turn-taking and social structure have indeed been explored, extensively at least in starlings (Henry et al., 2015), arguing for the potential co-evolution of turn-taking mechanisms and social structure; and in Japanese macaques (Arlet et al. 2015). In this latter study, the authors observe a correlation between the social bonds of some individuals (from their mutual grooming) and the frequency of their vocal exchanges, thus supporting the hypothesis that vocal exchanges can be interpreted as a form of 'grooming-at-a-distance'. They clearly draw a link with the notion of social cohesion:

"vocalizations replaced gestural grooming once it became impossible to allocate enough time to physically grooming all affiliated group members, as a form of 'vocal grooming-at-a-distance"” (Arlet et al., 2015)

Of the notions used in animal behavior literature, 'communication persistence' and 'response waiting' are also significant, creating a link between sequential mechanisms and cooperation. For instance, 'response waiting', defined as a signaler pausing for at least some seconds, while maintaining visual contact with the recipient, after having initiated an action, is interpreted as an expectation (Fröhlich et al., 2016a). This of course is not without raising thorny questions about the intentional dimension of communication. But here, as in human conversations, a noticeable absence of response is treated as a departure from the usual expectancies. Departures from the norm exhibit through contrast the norm-governing mechanism that is usually veiled when everything goes smoothly. Observations of all these behaviors enable animal behaviorists to make connections between sequential phenomena, expectancies, and goal-orientated communicational practices.

Non-human primates seem to remain the favored model for investigating the evolution of human language. This raises several issues, because despite frequent claims 
(for instance see Kendrick et al., 2020: "one of the most basic units of human social interaction [turn-taking, author's note] has apparent homologs in the social interaction of nonhuman primates" (2020: 134)), it is still unclear whether turn-taking mechanisms identified in non-human primates are indeed homologous or simply analogous (Pika et al., 2018). This is a critical question (Laland \& Brown, 2008). If they are analogous, they can serve as a heuristic metaphor to shed light on recurrences and matrixes. But if they are homologous, this assumes that there is an evolutionary continuity between rudimentary forms of alternation and more complex turn-taking sequences and rulegoverned conversational exchanges in humans. The idea that "great apes provide a window back in time on our last prelinguistic ancestor's communication and cognition" (Lameira \& Call, 2020) has been critically examined in several studies (Laland \& Brown, 2011; Leavens et al., 2017; Leavens, 2018; Lameira \& Call, 2020). Moreover, the turntaking capacities of birds such as ravens, parrots and starlings challenge the argument of phylogenetic proximity and allow for an additional hypothesis of 'functional convergence' (Henry et al., 2015).

All of the studies we have cited document observations related to rule-governed conversational structures, or turn-taking mechanisms, in the communicational repertoire of some animal species. We have examined how these findings are associated with hypotheses on the origins of human language and cooperation. These studies remain related to species-typical behaviors. In contrast, we would now like to make the case for studying interspecies interactions from a conversation analytical point of view, to explore what insights these might bring to the study of communication and social interaction in general.

\section{Why study interspecies interaction?}

It is one thing to observe "similarity between human conversations and communicatory interactions in non-human animals" (Logue \& Stivers, 2012, p. 1283), but a different approach is to look at how human and nonhuman animals communicate with each other, and see if these patterns are compatible - whatever the evolutionary explanations for compatibility or incompatibility may be.

I argue that two primary reasons justify a deeper look at interspecies interaction by analysts of social behavior:

- The first is that human-animal social interactions are a widespread phenomenon that has been largely under investigated in the field. The scarcity of studies interspecific interactions is as remarkable as the number of these interactions is great, in terms of presence in the households, but also as regards to the complexity of actions that can be accomplished in several "institutional" contexts (e.g. guide- or assistance-dog training).

- The second reason is that it offers a view upon rudimentary forms of interaction that are not completely amenable to an analysis in terms of cultural and linguistic rules; nor totally the product of species-typical ethological behaviors. In other words, our relationships with domestic animals are not fully understandable in functional terms (prey/predator, for instance) - and something much more complex in the adjustment of actions and in the subtleties of communicational exchanges is at stake. These interspecific rule-governed mechanisms are currently poorly understood.

Human social interaction has crucially been characterized by its reflexivity and accountability (Garfinkel \& Sacks, 1970). How do human-animal interactions work with regards to reflexivity, the question remains unsolved. What are the 'rules' - if they exist - in these hybrids (although perfectly ordinary) interactions, that are neither entirely governed by linguistic and cultural norms, nor completely the product of stereotypical species related behavioral patterns? 


\subsection{An overlooked area}

It is not the point here to engage in a comprehensive epistemological explanation for why interspecies interactions have been neglected in both linguistics and animal behavior scholarship. One part of the answer may lie in the interdisciplinary commitment required by this topic.

From the point of view of linguistics, the neglect of this issue may appear evident: language has been conceptualized as a human characteristic - even sometimes as the mark of human exceptionalism (Hauser, Chomsky, Fitch, 2002) - de facto excluding other animals. The controversy opposing the Dutch entomologist Von Frisch and the French linguist Benveniste at the beginning of 20th century is emblematic in that regard, with the former describing honeybee dance as a 'language', and the latter denying this label, considering it nothing more than a 'code of signals'. This position has long been interpreted as a consequence of the Cartesian tradition, considering animals (or 'beasts') as opposed to humans in their intellectual capacities. Animal studies have widely used this type of explanation to account for the reluctance in social sciences in general to deal with animals' actions, treated as mechanistic and instinctive behaviors, and therefore seen as irrelevant to any sociological inquiry (Crist, $1999 ; 2004)$.

In linguistics, the default model features a "prototypical competent speaker, fully endowed with all abilities required to engage in the process under study" (Goodwin, 2004, p. 151). In other words, what we could call logocentrism or, more broadly, the tropism towards articulated verbal language, has driven researchers to exclude forms of animal communication from their studies- let alone interspecies communication.

For all these reasons, and perhaps for many more, the study of human-animal communication has not been on the agenda in social sciences and linguistics (with the exception of a few studies which we discuss below). Likely, there is a presumption that treating animal behavior with the same analytical apparatus used with humans would cast over a potential shadow of reductionism.

In studies of animal behavior, on the other hand, scientific (and comparative) reasoning requires characterizing species-specific behavioral patterns. Almost by definition, ethological description is pointedly disinterested in interspecific interactions, except when they are described in terms of functional relations at a macro-level (however see Gacsi \& Miklosi, 2010).

Besides, there is minimal crossover between the disciplinary fields of linguistics and animal behavior to bridge the gap between studies in animal communication and human conversation analysis, with the exception of the original attempt by Logue \& Stivers (2012). The ambition of the authors is to import toolboxes from CA and they are very aware of the variability of the applicability of this model to a corpus of animal gestures or vocalizations. Despite their attempts of a cross-fertilization between CA and animal communication, it is not their point to address the cross-species dimension.

Lastly, even if more links were created between those two disciplines, this would not erase the deep theoretical and epistemological incompatibilities, that existing works using CA as a tool (Wilkinson, Leudar and Pika, 2012; Rossano \& Liebal, 2014) sometimes minimize. While the now famous paper by Sacks et al. (1974) is surprisingly very often cited in studies dealing with turn-taking mechanisms in ethology and animal social cognition (Henry et al., 2015; Fröhlich et al., 2016b; Fröhlich, 2017; Pika et al., 2018 , inter alia) and if conversation analytic - hence sociological - tradition remains mentioned, at least in the state of the art, this should not hide fundamental discrepancies in ways of understanding social order, and communication. For instance, finding out the biological basis of turn-taking mechanisms (or considering turn-taking as "simply part of our ethology" as Levinson (2016:10) puts it) was not overtly the goal pursued by Sacks, 
Schegloff and Jefferson. Despite a call for a naturalistic apprehension of human behavior formulated by Sacks (1992), it is on the contrary possible to argue that he had an antireductionnist view of human conduct (Lynch and Bogen, 1994; Schegloff, 1992, p. xxxii).

The differences in the apprehension of experimental setups, in the formulation of hypotheses on the evolution of language or human cooperation; and in the interpretation of interactions in evolutionary functional terms are crucial - as CA does not primarily draw on a biologically inspired framework.

\subsection{Some recent and seminal attempts to explore interspecies sequential} organization

Some works emanating from the social sciences, in symbolic interactionism (Arluke \& Sanders 1996; Sanders, 2003), sociolinguistics (Cornips, 2019), interactional linguistics (Tannen, 2004; Roberts, 2004), ethnomethodology (Goode, 2007; Laurier, Maze, Lundin, 2006), conversation analysis (MacMartin et al., 2014; Mondémé, 2011, 2018, 2019) have occasionally addressed the topic of interspecific interaction. The main point of interest in these studies for the topic at hand includes the analysis of human-animal interactions in terms of mutual adjustments, as they unfold sequentially.

Through their focus on the procedural and orderly aspect of human-animal interactions, ethnomethodological and CA approaches make it possible to avoid several impasses in classic sociological inquiry, regarding the (ontological) properties of agents or the intentional character of their behavior. During sequences of play between a dog and its owner (Goode, 2007) or during daily walks in parks (Laurier, Maze, Lundin, 2006), detailed and video-documented analyses show how "the display and recognition of actions from human to dog, and back and forth, similarly" can be treated (Laurier et al., 2006: 11). An ethnomethodological approach allows for a suspension of a cognitive conception of understanding. As Laurier et al. put it: "being able to access the "inner" experience of the other is not a requirement of being able to coordinate interaction with them. Moreover, those who have followed Sacks' (1992a, 1992b) studies of conversation and mind suspend the notion that individuals understand one another. Rather, there is demonstrable evidence that individuals have procedural competence in conducting joint actions" (Laurier et al. 2006, p. 5)

This statement has important consequences for the analysis of interspecies human-animal interactions: there is no need to adjudicate on the thorny problem of animal intentionality to observe the orderly accomplishment of complex joint actions - and to analyze them accordingly. This orderliness, although not explicitly described in terms of turn-taking in this literature, nevertheless sheds light on a type of sequential machinery, that has a clear analogy with the rules of a conversation:

"In some ways the machinery of play resembles conversation, in that it is sequentia and often involves an initiating action/utterance. The action/utterance constitutes the potential (...) boundary of play. Just as the conversation analysts write about the ways in which conversationalists open up conversational closings, or initiate openings to conversation, play also consists of the mutual production of signs and invitations to begin (and later to end) play. (Goode, 2007, p. 50)

Since the 2000s, a series of articles published in Research on Language and Social Interaction have focused on ordinary interactions involving pet animals, in families (Tannen, 2004) or in veterinary clinics (Roberts, 2004). These two 2004 articles highlight the role played by animals as resources in the management of interpersonal human interactions. Ten years later, MacMartin, Coe and Adams (2014) elaborate on the previous 'vet-to-pet' studies, showing how animals, in the veterinary context, are treated as interactional participants. These authors describe a sequential format, where a response token "I know" follows an animal's visible display of distress elicited by the medical activity. Methodologically, animals feature in the transcript and are being assigned a participant status, alongside humans. In accordance with the conversationalist's claim, 
the methodological apparatus thus reflects the participant's orientations to pets as being interactionally competent beings.

Drawing on this body of work, a further set of studies (Mondémé, 2018, 2019, 2020) provides sequential analyses of dog-human interactions, both in the institutional context of guide-dog training and in the domestic context of family households. These studies shed light on different formats in which dogs initiate actions that are embedded in complex sequential formats. A growing body of research in this area is emerging, although it is still in its infancy and has only been presented at conferences for the moment (e.g. Deppermann, 2017 ; Harjunpää, in press)

Yet the scope of this small number of existing studies is limited. No works so far have carried out a systematic examination of interspecies interactions through the lenses of sequential analysis.

\section{Sequence organization and turn-taking in interspecies interaction:} limits and programmatic developments

The analysis of interactions involving animals requires avoiding certain pitfalls. I shall briefly mention them before turning to a discussion on the limits of the analytical notion of 'turn' when applied to animal's actions.

There are two main difficulties, which have to do with the interpretation of the animal's behavior within a sequence: how can we know for sure that what we label e.g. an addressed gaze is not a pure coincidence? Is it not too daring, or too anthropomorphic, to consider this particular behavior as a 'request'? These issues pertain to positions referred to as, in a broad outline, behavioristic (i) and intentionalist (ii)

- A behavioristic position would argue that an animals' actions are only reactions, oriented to a given stimulus (a vocal order for instance), and that in lieu of accounting for the complex and reflexive dimension of mutual adjustment at the heart of social interaction, it only points to the previous conditioning of the animal. While this interpretation cannot be completely excluded, it does not seem satisfactory to fully explain the mechanisms of social interaction. The ontogenetic and socializing process experienced by a domestic animal cannot be reduced to a succession of consolidated stimuli/responses. As a methodological proviso, it seems justifiable to suspend the idea that an animal who was socialized to and in the human world would be a Cartesian machine able only to react, and to borrow Lynch's words (2007), "to grant that the nonhuman 'other' of the encounter is an agent rather than a mechanism that can only react on cue" (Lynch, 2007, p. xiv);

- The second pitfall relates to the ascription of unwarranted intentions to animals' actions. The ethnomethodological position offers, as pointed out earlier, a way out. Suspending the idea, generally assumed in symbolic interactionist approaches, that shared understanding is required to accomplish an action - even within human interactions allows interspecies interactions to be considered without having to speculate on the degree of agreement, or mind reading, shared by the two parties.

Both positions can be addressed by the notion of "next turn proof procedure" (Schegloff, 2007), on the condition of treating the animal's action as a 'turn' on its own, which is discussed later.

\subsection{Working out the core concepts of CA: identifying sequential formats in} human-animal interactions?

Over the last ten years, several hours of video recordings taken in different institutional and domestic contexts involving domestic as well as wild species interacting with humans have allowed the observation of the systematicity of actual sequential formats. These include, for instance: (i) assessment sequences, triggered by a dog's addressed gaze directed to the human participant (Mondémé, 2019); (ii) sequences of strokes elicited by 
a horse or a pet, via diverse embodied resources (Mondémé, 2020); (iii) more expectedly, sequences of request and offer, especially revolving around food issues, with wild animals (Barbary macaques and tourists in Morocco) as well as with domestic pets, in the ordinary life of Western households. These three cases do not claim to be exhaustive, and are by no means representative of the diversity and the variety of (sometimes) complex humananimal interactions. Yet the identification of such sequential formats represents a stepping stone for future research in the systematicity of actual instances of human-animal sequences of interaction, and surely deserves further investigation.

These sequences, situated in larger courses of action, "are the vehicle for getting [a certain] activity accomplished" (Schegloff, 2007: 59). The fact that the action performed by the human (e.g. an assessment, a stroke, an offer) 'follows' the animal's action appears to indicate sequential unfolding that carries normative expectancies - not just due to the frequency and recurrence of the format, but mainly because when the expected receipt is not produced by the human participant, this absence is made noticeable by the animal through diverse procedures such as repairs or expansions of the sequence.

Our deliberated focus on animals' initiations of action, namely here actions that could be considered a first-pair part, avoids a too narrow behavioristic understanding of the phenomenon, which would view the animal as simply reacting to a stimulus through habituation or conditioning. In fact, animals often initiate sequences of actions, relying on multimodal resources, by means of vocalization (growling, meowing) or embodied actions of diverse kinds (hand gestures in monkeys, paw striking, or snout nudging in domestic dogs, head and body orientation in horses, as well as more subtle multimodal resources, such as addressed gazes) that play the role of turn allocational components.

An examination of these formats reveals systematicities in the distribution of action that seems to bridge species barriers. This may be the result of enculturation or socialization or whatever we might call this process of living with one another for years, including over the longer evolutionary scale. Whatever the reason, the result seems to be that humans and animals are able to engage in joint communication and accomplish very complex cooperative actions. There appear to be mechanisms - whatever their evolutionary function - that allow for interspecies communication, beyond or below the ethological rules that supposedly govern behavior. While we know this from ordinary experience, the analytical demonstration of sequential formats with adjustments made by each party, resembling the machinery described in human interactions, is intriguing and shows that this area merits further investigation.

The identification of typical formats is interesting not only because it advances our understanding of different modalities of interaction with animals' species we live or mix with. It also reveals the expectancies that are mutually produced. As in human interactions, a departure from a given sequential format (a noticeable absence after a summons, for instance) is highly significant and, through contrast, reveals the normative and moral dimensions that also underlie human-animal interactions.

\subsection{Do animals 'take turns'?}

In CA approaches, a 'turn' is an analytical category described as such by virtue of its relationship with the previous and to the next action or utterance of a co-participant. As seminally defined by Sacks et al. (1974):

"The turn-taking system has, as a by-product of its design, a proof procedure for the analysis of turns. B [the recipient, $n / a$ ] (...) thereby displays (in the first place to his co- participants) his understanding of the prior turn's talk as a first part, as a "question" or "complaint" (...) It is a systematic consequence of the turn-taking organization of conversation that it obliges its participants to display to each other, in a turn's talk, their understanding of other turns' talk" (Sacks et al., 1974, p. 44) 
The notion of 'understanding' here is crucial, and has recently led ethnomethodology and conversation analysis (EMCA) researchers to develop an embodied, sequential conception of understanding - a perspective, according to Mondada (2011) that originated in Ryle and Wittgenstein. Here 'understanding' is not to be viewed as an operation applying to mental states, but to the meaning of conduct (Sidnell, 2015, p. 372, see also Lynch, 2011; Lindwall \& Lymer, 2011; Broth, Cromdal, Levin, 2019, for related remarks). In line with this sequential comprehension of understanding, any production of meaning in a sequence of action that is treated as such by the participants could be pragmatically considered a turn, in spite of its non-sentential, non-clausal or non-lexical form. 'Pragmatically considered' does not mean that it is a turn, but that it might be considered to have the same functional relevance.

In the context of interspecies interactions, it is critical to unpack this issue: before knowing whether an animal can produce turns, we need to settle on whether any nonverbal or embodied action can be considered a 'turn' at all. Despite an increasing body of work on embodied interaction (Nevile, 2015) in the field, the idea that a turn would not take the shape of a turn's talk but would be exclusively embodied - and therefore not bear any clear propositional content - is not self-evident. This question has been extensively discussed over the past 20 years in the literature: for example, the concept of "multimodal utterance" (Goodwin, 2006; Goodwin, Streeck, Lebaron, 2011) in particular, is one of the many attempts to settle a terminology. But this term, generally used to refer to hybrid forms mixing non-verbal utterances with non-verbal completions (like e.g. pointing), has been critically discussed by Keevallik (2013):

"since uttering involves speech, the term utterance may not be the best one to characterize instances when speech is not clearly profiled. It may convey the assumption that speech is somehow still most crucial for communication" (Keevallik, 2013: 2).
It is clear that the call to examine sequences of interspecies interaction rests on the analytic assumption that it is possible to analyze sequences of paired action that involve at least "silent embodied actions" (Mondada, 2019). In human social interaction, this has been studied in the context of an embodied action in response to a request (e.g. Goodwin \& Cekaite, 2014; Mondada, 2017, 2019), thus operating as a 'bodily implemented' second pair part (Mondada, 2019). More recently, several "non-talk-based forms of social organization" (Ivarsson \& Greiffenhagen, 2015) - especially social activities such as sports and leisure - have been described as featuring embodied turntaking practices: for example, in surfing (Liberman, 2015), skating (Ivarsson \& Greiffenhagen, 2015), or dancing (Keevallik \& Ekström, 2019). Drawing on these approaches, we argue that, although it is not unproblematic, it is possible and heuristic, to take an analytical premise that considers an animal's actions as turns as long as they are oriented to, and understood, by human participants, as meaningful behaviors inserted into a relevant sequence of actions.

This conversational machinery featuring sometimes verbal, sometimes purely embodied segments, is clearly described by Goode (2007), in references to sequences of play with his own dog:

"The way it worked has something to do with the ways dogs participate in conversation. I talked to Katie in the course of the play to exhort her to action, to tell her what action I wanted her to do, to ask her how she was feeling or if she was hurt, etc. Her 'answer', her turn of talk, was made in the form of behavior. Talk was thus meaningfully embedded in the unfolding, in situ course of mutually produced bodily events." (Goode, 2007: 78; [my emphasis])

Here the dog is not literally taking her turn through utterances, but through active bodily participation. From his previous work on interactions between severely disabled children and her caretakers (Goode, 1994), Goode elaborates an argument about the rich and 
orderly social relationship that can exist without shared (linguistic) symbols. Although the two contexts are radically different, and are in many ways "incomparable" (Goode, 2007: 130), he extends this reasoning, and his "general model for intersubjectivity" (2007: 89) to the analysis of interspecies play. Making sense of animal behavior and action is therefore achieved through ordinary perception, what Goode's calls the "vulgar availability of Katie's request to play" (2007: 50) originated in the fact that, to a certain extent, different species can share a common Umwelt.

\section{Conclusion}

$$
\begin{aligned}
& \text { "You can't have meaningful communication without grammar- } \\
& \text { without a structure that is embedded in time. Even in the case of what } \\
& \text { appears to be a gesture or signal consisting of a single counter, as when } \\
& \text { Belle barks to be let out to run, the bark or the tail-wagging or door- } \\
& \text { scratching is meaningless without what goes on before and after coming } \\
& \text { in certain ways, in a rule-governed sequence » (Hearne, 1986, p. } 97 \text { ) }
\end{aligned}
$$

This article has examined recent studies which identify turn-taking mechanisms in rulegoverned vocal or gestural alternations in several animal taxa. It argues that analyzing action in terms of turn-taking should analytically show more than the strict alternation of phylogenetically ritualized sequences.

This article makes the case for going beyond the study of only intraspecific forms of communication and interaction. What does an analysis of interspecies interaction allow us to see that cannot be observed in species-specific animal interactions? Equally, what does an analysis of interspecies interaction allow us to see that an observation of only human interaction may hinder?

Animal behaviorists reason at the level of species. This directly implies that behaviors, even communicative behaviors, are part of the species' ethogram, or repertoire. Although communicative behaviors have been reported to be context-sensitive in many social species, they are interpreted as stereotypical displays with specific evolutionary functions. In that regard, human turn-taking could be considered a display, within the human communicational repertoire, of stereotypical forms of communication specific to Homo sapiens, a display that is shared in some of its basic aspects with several other social species. This proposition naturalizes communicational phenomena;

On the other hand, in the EMCA tradition, the turn-taking argument is conceived as stemming from an important sociological background (borrowing from phenomenology, with a pragmatist bent) that defends a more complex conception of “turn-taking”, as driven by conditional relevance and normativity. Sacks' Lectures argued that the orderliness of human actions is amenable to systematic study - as does the naturalistic study of animal behavior. However, whereas the regularity of animal behavior is described by ethologists as a sign of the typicality of species' behavior, human systematic orderliness is viewed as a reflexive process allowing for the accomplishment of cooperative social actions.

These conceptual and disciplinary distinctions are challenged when considering human-animal interactions. A reductionist ethogram-based approach is obviously unsatisfactory to make sense of the richness involved in coordinated actions between a pet and its owner (to put it crudely, humans do not bare their teeth or wag their tails when interacting with dogs). One does not have to assume either phylogenetical inheritance, or a hierarchical ascendance based on the mastery of articulated language, to observe efficient - and sometimes complex - forms of interspecies communication. These interactions exhibit systematic features that suggest that it may be a worthy endeavor to broaden the concept of the 'interaction engine' (Levinson, 2006) - which by definition sets human communication apart from the communicative system of other animals - and extend it to interspecies communication and sociality.

\section{References}


Altmann, S. A. (1965). Sociobiology of rhesus monkeys. II: Stochastics of social communication. Journal of Theoretical Biology, 8(3), 490-522.

Arlet, M., Jubin, R., Masataka, N. \& Lemasson, A. (2015). Grooming-at-a-distance by exchanging calls in non-human primates. Biol. Lett. 1120150711

Arluke, A., \& Sanders, C. (1996). Regarding Animals. Animals, culture, and society. Philadelphia: Temple University press.

Boyd, R., Richerson, P. (1985). Culture and the Evolutionary Process, Chicago: The University of Chicago press.

Broth M., Cromdal, J., Levin, L. (2019). Telling the Other's side: Formulating others' mental states in driver training, Language and Communication, 65, 7-21.

Burkhardt, R. (2005). Patterns of Behavior: Konrad Lorenz, Niko Tinbergen, and the Founding of Ethology. Chicago University Press

Button, G, Sharrock, W. (2016). In support of conversation analysis' radical agenda. Discourse Studies, 18(5), 610-620.

Cornips, L. (2019). The final frontier: non-human animals on the linguistic research agenda. In J. Berns \& E. Tribushinina (Eds.), Linguistic in the Netherlands (pp. 13-19). Amsterdam: John Benjamins.

Crist, E. (1999). Images of Animals: Anthropomorphism and Animal Mind. Philadelphia: Temple University Press.

Crist, E. (2004). Can an insect speak? The case of the honeybee dance language, Social Studies of Science, 34(1), 7-43.

Demartsev, V., Strandburg-Peshkin, A., Ruffner, M. \& Manser, M. (2018). Vocal turntaking in meerkat group calling sessions. Current Biology, 28, 3661-3666.

Deppermann, A. (2017). On the integration of natural order into social order: the case of bird of prey demonstrations, Communication at the 15 th International Pragmatics Conference, Belfast, Northern Ireland, 20th July 2017.

Deputte, B.L. (1982). Duetting in male and female songs of the white-cheeked gibbon (Hylobates concalor lrucogenys), in C.T. Snowdon, C.H. Brown, \& M.R Petersen, (Eds.) Primate Communication (pp 67-93). Cambridge University Press.

Duncan, S. (1972). Some signals and rules for taking speaking turns in conversations. Journal of Personality and Social Psychology, 23(2), 283-292.
Duncan, S. \& Fiske, D. (1979) Dynamic Patterning in Conversation: Language, paralinguistic sounds, intonation, facial expressions, and gestures combine to form the detailed structure and strategy of face-to-face interactions, American Scientist, Vol. 67, No. 1, 90-98.

Duranti, A., Goodwin, C. (Eds.). (1992). Rethinking Context: Language as an Interactive Phenomenon. Cambridge University Press.

Fedurek, P., \& Slocombe, K. E. (2011). Primate vocal communication: a useful tool for understanding human speech and language evolution? Human Biology, 83(2), 153-173.

Fröhlich, M.; Wittig, R., Pika, S. (2016a). Should I stay or should I go? Initiation of joint travel in mother-infant dyads of two chimpanzee communities in the wild. Animal Cognition, 19(3), 483-500.

Fröhlich, M., Kuchenbuch P., Müller, G., Fruth, B., Furuichi, T., Wittig, R. \& Pika, S. (2016b). Unpeeling the layers of language: Bonobos and chimpanzees engage in cooperative turn-taking sequences. Scientific Reports, 6, 1-14. DOI: $10.1038 /$ srep258871

Fröhlich, M. (2017). Taking turns across channels: Conversation-analytic tools in animal communication. Neuroscience \& Biobehavioral Reviews, 80, 201-209.

Gacsi M., Miklosi, A. (2010). Human-animal interaction (Special Issue), Interaction Studies, 11(3), 349-352.

Garfinkel, H, \& Sacks, H, (1970). On formal structures of practical action. In J. C. McKinney \& E. A. Tiryakian (Eds.), Theoretical sociology: perspectives and developments (pp. 338-66). New York: Appleton-Century-Crofts.

Goffman, E. (1964). The neglected situation. American anthropologist, 66(6), 133-136. Goffman, E. (1981). Forms of talk. University of Pennsylvania Press

Goode, D. (1994). A world without words: The social construction of children born deafblind. Philadelphia: Temple University Press

Goode, D. (2007). Playing with my Dog Katie. An Ethnomethodological Study of DogHuman Interaction. Purdue University Press.

Goodwin, C. (2004). A competent speaker who can't speak: the social life of aphasia. J. Linguist. Anthropol. 14 (2), 151-170

Goodwin, C. (2006). Retrospective and Prospective Orientation in the Construction of Argumentative Moves, Text and Talk. 26(4/5), pp. 443-461. 
Goodwin, M. \& Cekaite, A. (2014). Orchestrating directive trajectories in communicative projects in family interaction. In P. Drew \& E. Couper-Kuhlen (Eds.) Requesting in social interaction (p. 181-210). Amsterdam: Benjamins.

Hauser, M., Chomsky, N., Fitch, T. (2002) « The Faculty of Language: What Is It, Who Has It, and How Did It Evolve? », Science, Vol. 298, Issue 5598, 1569-1579.

Hearne V. (1986). Adam's Task: Calling Animals by Name. New York: Vintage Books. Henry, L., Craig, A. J. F. K., Lemasson, A. \& Hausberger, M. (2015) Social coordination in animal vocal interactions. Is there any evidence of turn-taking? The starling as an animal model. Front. Psychol. 6, 1416.

Heritage, J. (1984). Garfinkel and Ethnomethodology. Cambridge: Polity Press.

Hutchby I. (1995). Aspects of Recipient Design in Expert Advice-Giving on Call In Radio, Discourse Processes, 19(2), 219-238.

Ivarsson, J., \& Greiffenhagen, C. (2015). The Organisation of Turn-Taking in Pool Skate Sessions, Research on Language and Social Interaction 48 (4), 406-429.

Keevallik, L. (2013). The Interdependence of Bodily Demonstrations and Clausal Syntax, Research on Language \& Social Interaction, 46:1, 1-21

Keevallik, L. \& Ekström, A. (2019). How to Take the Floor as a Couple: Turn-Taking in Lindy Hop Jam Circles. Visual Anthropology, 32(5), 423-444.

Kendrick, K. H., Brown, P., Dingemanse, M., Floyd, S., Gipper, S., Hayano, K., ... \& Levinson, S. C. (2020). Sequence organization: A universal infrastructure for social action. Journal of Pragmatics, 168, 119-138.

Laland, K., Brown, G. (2008) Comments on «The Chimpanze Has No Clothes» by Sayers \& Lovejoy, Current Anthropology, 49, 101-102.

Laland, K., Brown, G. (2011). Sense and Nonsense: Evolutionary Perspectives on Human Behaviour. Oxford University Press.

Lameira, A., Call, J. (2020). «Understanding Language Evolution: Beyond PanCentrism ». BioEssays, 42, https://doi.org/10.1002/bies.201900102

Laurier, E., Maze, R., Lundin, J. (2006). Putting the dog back in the park: Animal and human mind-in-action, Mind, Culture and Activity, 13, 1, 2-24.

Leavens, D., Bart, K., Hopkins, W. (2017). The Mismeasure of Ape Social Cognition, Animal Cognition, 22 (4), 487-504.

Leavens D. (2018). " The Cognitive Implications of Intentional Communication: A Multifaceted Mirror ». In Di Paolo, L., Di Vincenzo, F., De Petrillo F. (Eds.), Evolution of Primate Social Cognition (pp. 59-77). Springer Verlag.
Levinson, S. (2006). On the human "interaction engine". In: N. Enfield \& S. Levinson, (Eds.), Roots of Human Sociality: Culture, Cognition and Interaction (pp. 39-69). Berg, Oxford.

Levinson, S. (2016). Turn-taking in human communication - origins and implications for language processing. Trends in Cognitive Sciences 20, 6-14.

Levinson, S., Holler, J. (2014). The origin of human multi-modal communication. Phil. Trans. Biol. Sci. 369, 20130302. https://doi.org/10.1098/ rstb.2013.0302.

Lévrero, F., Touitou, S., Fredet, J., Nairaud, B., Guery, J. P. \& Lemasson, A. (2019). Social bonding drives vocal exchanges in bonobos. Scientific Reports 9(1), 711

Liberman, K. (2015). “Turn-Taking in the Surfers’ Lineup.” Surfer Today, October 16. https://www.surfertoday.com/surfing/turn-taking-in-the-surfers-lineup-anacademic-analysis-by-kenneth-liberman

Liebal K., Call J., Tomasello M. (2004). Use of gesture sequences in chimpanzees, American Journal of Primatology, 64, 377-396.

Lindwall, O., Lymer, G., 2011. Uses of "understand" in science education. Pragmatics 43, 452-474.

Logue, D., \& Stivers, T. (2012). Squawk in interaction: a primer of conversation analysis for students of animal communication. Behaviour 149, 1283-1298.

Logue D., \& Krupp D. (2016). Duetting as a Collective Behavior, Frontiers in Ecology and Evolution, 4, 7

Lynch, M. \& Bogen, D. (1994). Harvey Sacks' Primitive Natural Science, Theory, Culture \& Society, vol. 11, n 4, 65-104

Lynch, M. (2007) Introduction, in D. Goode, Playing with my Dog Katie An Ethnomethodological Study of Dog-Human Interaction, (pp. xiii-xv). Purdue University Press

MacMartin, C., Coe, J., Adams, C. (2014). Treating distressed animals as participants: I know responses in veterinarians' pet-directed talk, Research on Language and Social Interaction, 47, 2, 151-174.

Masataka, N. (2003). The Onset of Language, Edition (Volume 9). Cambridge University Press.

Mondada, L. (2011). Understanding as an embodied, situated and sequential achievement in interaction, Journal of Pragmatics, 43 (2), 542-552. 
Mondada, L. (2019). Transcribing silent actions: a multimodal approach of sequence organization. Social Interaction. Video-Based Studies of Human Sociality. 2:2. DOI: 10.7146/si.v2i1.110964

Mondémé, C. (2011). Animal as subject matter for social sciences: when linguistics addresses the issue of dog's "speakership" ", in Gibas P. Pauknerova K., Stella M. (Eds.). Non-humans in Social: Science: Animals, Spaces, Things. (pp. 87-104). Cerveny Kostelec: Pavel Mervart.

Mondémé, C. (2018). How do we talk to animals? Modes and pragmatic effects of communication with pets, Langage et société, no 163(1), 77-99. https://doi.org/10.3917/ls.163.0077

Mondémé, C. (2019). La socialité interspécifique. Pour une analyse multimodale des interactions hommes-chiens. Lambert-Lucas.

Mondémé, C. (2020). Touching and petting: exploring "haptic sociality" in interspecies interaction, in A. Cekaite. \& L. Mondada L. (Eds), Touch in social interaction. Touching moments. Routeledge.

Nevile, M. (2015). The Embodied Turn in Research on Language and Social Interaction, Research on Language and Social Interaction, 48(2), 121-151.

Pedersen, J. (2016). Apes in conversation: The role of the human interlocutor, Language \& Communication, 50, 1-11.

Pika, S., Wilkinson, R., Kendrick, K., Vernes S. (2018). Taking turns: bridging the gap between human and animal communication. Proceedings of the Royal Society. B 285: 20180598.

Pougnault, L., Levréro, F., Mulot, B. \& Lemasson, A. (2020). Breaking conversational rules matters to captive gorillas: A playback experiment. Scientific Reports, 10(1), $1-10$.

Premack D., Woodruff G. (1978). Does the chimpanzee have a theory of mind? Behavioral and Brain Sciences, vol 1 (4), 515-526.

Prieur, J., Barbu, S., Blois-Heulin, C., \& Lemasson, A. (2020). The origins of gestures and language: history, current advances and proposed theories. Biological Reviews, 95(3), 531-554

Richerson, P. \& Boyd, R. (1998). The Evolution of human ultra-sociality, in I. EiblEibisfeldt \& F. Salter (Eds.), Indoctrinability, Ideology, and Warfare: evolutionary perspectives. (pp. 71-95). New York: Berghahn Books.
Roberts, F. (2004). Speaking to and for animals in a veterinary clinic: A practice for managing interpersonal interaction, Research on Language and Social Interaction 37(4), 421-446.

Rossano, F. (2013). « Sequence organization and timing of bonobo mother-infant interactions », Interaction Studies, 14 (2), 160-189.

Rossano, F. \& Liebal, K. (2014). Requests" and "offers" in orangutans and human infants in P. Drew \& E. Couper-Kuhlen, Requesting in Social Interaction (pp. 333-362). Amsterdam: Benjamins.

Sacks, H., Schegloff, E. \& Jefferson, G. (1974). A simplest systematics for the organization of turn-taking for conversation, Language, 50, 696-735.

Sacks, H. (1992). Lectures on Conversation. Vol 1 \& 2. London: Basil Blackwell.

Sanders, C. (2003). Action speak louder than words: close relationship between humans and non-human animals. Symbolic Interaction, 26: 3, 405-426.

Schegloff, E. A. (1972). Notes on a conversational practice: Formulating place. In D. Sudnow (Ed.), Studies in social interaction (pp. 75-119). New York: Free Press.

Schegloff, E. A. (1992). Introduction, in H. Sacks, Lectures in Conversation, vol. 1 (p. ix to xii). Oxford: Blackwell.

Schegloff, E. A. (2007). Sequence organization in interaction: A primer in conversation analysis (Vol. 1). Cambridge, England: Cambridge University Press

Schlenker, P., Chemla, E., \& Zuberbühler K. (2017). Semantics and Pragmatics of Monkey Communication, in Aronoff, M. (Ed.), Oxford Research Encyclopedia of Linguistics. New York, Oxford University Press.

Sidnell, J. (2015). The architecture of intersubjectivity revisited. In: Enfield, N.J., Kockelman, P., Sidnell, J. (Eds.), Cambridge Handbook of Linguistic Anthropology. Cambridge University Press.

Slocombe, K. E., Waller, B. M., \& Liebal, K. (2011). The language void: the need for multimodality in primate communication research, Animal Behaviour, 81(5), 919924

Snowdon, C. T. \& Cleveland, J. (1984). "Conversations" among pygmy marmosets. American Journal of Primatology, 7, 15-20.

Stivers, T., Enfield, N., Brown, P., Englert, C., Hayashi, M., Heinemann, T., Hoymann, G., Rossano, F., de Ruiter, J., Yoon, K-E., \& Levinson, S. (2009). Universals and Cultural Variation in Turn-Taking in Conversation. Proceedings of the National Academy of Sciences 106(26): 10587-92. 
Streeck J., Goodwin C., LeBaron C. (eds.). (2011). Embodied interaction: Language and body in the material world. Cambridge University Press.

Tannen, D. (2004). Talking the dog: Framing pets as interaction resources in family discourses, Research on Language and Social Interaction 37(4), 399-420.

Todt, D., \& Naguib, M. (2000). Vocal interactions in birds: the use of song as a model in communication, Advances in the Study of Behavior, 29, 247-296.

Wilkinson, R., Leudar, I., Pika, S. (2012). Requesting behaviours within episodes of active sharing: A new look on chimpanzee signaling, in S. Pika \& K. Liebal (Eds.) Developments in Primate Gesture Research (pp.199-222). John Benjamins.

Wimmer, H., \& Perner, J. (1983). Beliefs about beliefs: Representation and constraining function of wrong beliefs in young children's understanding of deception. Cognition. 13, 103-128.

Zuberbühler, K. (2005). The phylogenetic roots of language: Evidence from primate communication and cognition. Curr. Dir. Psychol. Sci. 14, 126-130. 